\title{
Proceeding
}

\section{Muscle stimulation timing while implementing Ura Mawashi Geri in Iranian elite women}

\author{
SOMAYEH KHANZADEH ${ }^{* 1}$, HEIDAR SADEGHI2, SAJAD KARIMI CHOGHAGALANI ${ }^{1}$, SUSAN \\ HOSEIYNPOUR ${ }^{3}$ \\ ${ }^{1}$ Biomechanics, MA, Islamic Azad University of Tehran, Iran \\ ${ }^{2}$ Full Professor of Kharazmi University, Tehran, Iran \\ ${ }^{3}$ Physical Education, MA, Payam Noor University of Tehran, Iran
}

\begin{abstract}
Khanzadeh, S., Sadeghi, H., Choghagalani, S.K., \& Hoseiynpour, S. (2015). Muscle stimulation timing while implementing Ura Mawashi Geri in Iranian elite women. J. Hum. Sport Exerc., 9(Proc2), pp.S677S686. Introduction: Karate is a martial art in which the technics are performed by kicks, punches (hand) and rotational movements. Ura MAWASHI GERI is a kind of kick which involves the core stability muscles of Lumbar - Pelvic areas, Quadriceps muscle (Lower extremity) and knee and lumbar - pelvic joints while being performed. The purpose of this research is to determine the muscle stimulation time pattern of Gluteus Medius (GM), External Oblique (EO) and Quadriceps muscles such as Vastus Medialis (VM), Vastus Lateralis $(\mathrm{VL})$ at any phases of the implementation of the technique by the dominant leg. The muscular activities of VM, VL, EO and GM in 5 Iranian healthy elite Karatekas (in average age of (21/4) were recorded by the Electromyography device. The onset latency of the muscle activity and the Goniometric data (Knee Joint) were defined after processing the RMS of data and according to the Mean+4sd. After that, the average of the obtained records was used to determine the onset of each muscle separately at all phases of the implementation of the techniques. The results showed Glutesus Medius, at the phases of one, four, and five, and Vatus Medialis, at the phases of two and six, and the External Oblique at the third phase act earlier in comparison with other muscles. Key words: KARATE, URA MAWASHI GERI, ELECTROMYOGRAPHY, MUSCLE TIMING.
\end{abstract}

\footnotetext{
Corresponding author. Islamic Azad University of Tehran, Iran

E-mail: Somayiehkhanzadeh@yahoo.com

Asia Pacific Conference on Performance Analysis of Sport, 21-24 April 2014. Langkawi, Malaysia.

JOURNAL OF HUMAN SPORT \& EXERCISE ISSN 1988-5202

(c) Faculty of Education. University of Alicante

doi:10.14198/jhse.2015.10.Proc2.06
} 


\section{INTRODUCTION}

Karate is regarded as one the most popular martial arts. The word Karate is made of two words of Kara (empty) and Te (hand), which means fighting with empty hands. In other words, in Karate, one uses different technics of kicks (leg) and punches (hand) and defends without the using of any firearms. It is practiced in two fields of KATA (practice of a collection of techniques against no direct fighters), and KUMITE (fighting against real opponents) (Critchley \& Meredith, 1999; Emmermacher et al., 2007; Witte et al., 2007). Techniques like URA MAWASHI GERI, UKO GERI KEAGE, GER KEAGE, YOKO GERI KEKOMI, MAE GERI and MAWASHI GERI and etc., are performed by leg. URA MAWASHI GERI is one of the most important and highly used technics by the athletes in KUMITE which can result in high scores (Earl et al., 2001).

Karate, as a physical skill and art, is an active and dynamic sport which involves various muscles when performing the technics such as Ura Mawashi Geri. These techniques cause Flexion, extension, abduction, rotation ... in knee joints, thigh and lumbar- pelvic areas (Mellor, 2005; MacGregor et al., 2005; Stensdotter et al., 2008; Wong, 2006). Correct and appropriate use of the muscles and coordination between the movements of the legs and pelvis play a crucial role in the correct implementation of Ura Mawashi Geri (Mahmoud \& El-Daly, 2010; Antakly, 2010). Core lumbar stability muscles, Lumbar - pelvic and quadriceps muscles are of the most important and major muscles of the trunk and lower extremities which play an important role in knee joints, lumbar pelvic areas and postural control as the stabilizer (Mellor, 2005; MacGregor et al., 2005; Stensdotter et al., 2008; Wong, 2006; Leinonen, 2013).

Among the four parts of the quadriceps $(\mathrm{VM})$ and $(\mathrm{VL})$ are of great importance as the lack of coordination of these two muscles can cause knee injuries (MacGregor et al., 2005; Stensdotter et al., 2008; Wong, 2006; Coqueiro et al., 2005; Cavazzuti et al., 2010; Herrington \& Pearson, 2006; Herrington, et al., 2006; Ivkovic et al., 2007; Jafari et al., 2003). And among the core stability muscles of the Lumbar - pelvic area, the (GM) and (EO) have a significant role in stabling Patella and postural joint of lumbar - pelvic areas (Akuthota et al., 2008; Baffal et al., 2012; Sarabon et al., 2010).

There were different studies dealing with the onset latency of muscle activity of VM, VL, GM and EO when performing various activities, such as scout, going up the stairs and mountain climbing (Coqueiro et al., 2005; Cavazzuti et al., 2010; Herrington \& Pearson, 2006; Herrington, et al., 2006; Ivkovic et al., 2007; Jafari et al., 2003; Sarabon et al., 2010; Wong, 2009; Lederman, 2010). While some other researches focus on the effects of different angels of the joints' moving territory, and the effects of different kinds of balance on the onset latency of the muscles of VM, VL and GM (Dixon \& Howe, 2007; Ebrahimi \& Hafezi, 2000). But all of these researches were done on athletes with injuries (anterior knee and lower back pain) only, or in comparison with healthy athletes.

The present study tries to determine the muscle stimulation time pattern of VM, VL, GM and EO muscles while performing Ura Mawashi Geri separately at all of its implementation phases. We hope the results of the this study help identify the major involving muscles at each related phase to provide appropriate fitness programs to describe the dynamic motion of the technique at each phase, for the precise and right implementation of the techniques. 


\section{METHODOLOGY}

In this quasi-experimental study, there were 5 Iranian female KARATEKAS with World and Asian titles in the last 4 years participating ( 5 healthy women with the variance and standard deviation age of $21 / 4 \pm 3 / 9$, height of $166 / 8 \pm 4 / 2 \mathrm{~cm}$, Weight of $60 / 2 \pm 3 / 1$ with the $7 / 2 \pm 4 / 8$ years of professional experience in Karate). The Measuring tools used in the survey were personal and athletic information questionnaire of the of the participants, the examining the injuries related to the lower limb questionnaire (obtained by the American Orthopedic Association), and Electromyography device (ME 6000, Sampling rate $=1000 \mathrm{~Hz}$, Channel=16, Data transfer=walan, made in FINLAND) for recording the signals produced by the VM, VL, GM and EO muscles when performing Ura Mawashi Geri technic ,and also Electrogoniometer (Mega win , FINLAND)in order to define the dynamic range of the knee joint while implementing this technic.

To determine the position of the Chest Leads and Electrogoniometer, we first shaved the skins and cleaned it with alcohol in order to reduce the impedance of the electrode and the skin. After the skin was cleaned with alcohol, we next cleaned it by soft sanders. The Chest Leads used in this test were oval- shaped and of F-521L type and were attached to the specified place on skins with the spacing of $3 \mathrm{~cm}$ from each other (distance of center to center).

The Chest Leads were made of silver-chloride and were placed according to the instruction of SENIAM electrode attachment to muscles of $\mathrm{VL}$ (at an angle of 15 degrees and $10 \mathrm{~cm}$ above the upper edge of the outer patella and the muscular fibers), VM (at an angle of 50 degrees and $4 \mathrm{~cm}$ above the upper edge of the patella and in the direction of the muscular fibers), GM (1/3 spacing between the upper iliac crest and the greater tuberosity of femur), and EO (1/2 spacing between the twelfth rib and Asis with the angle of 30 45 degrees $\left.^{\circ}\right)$.
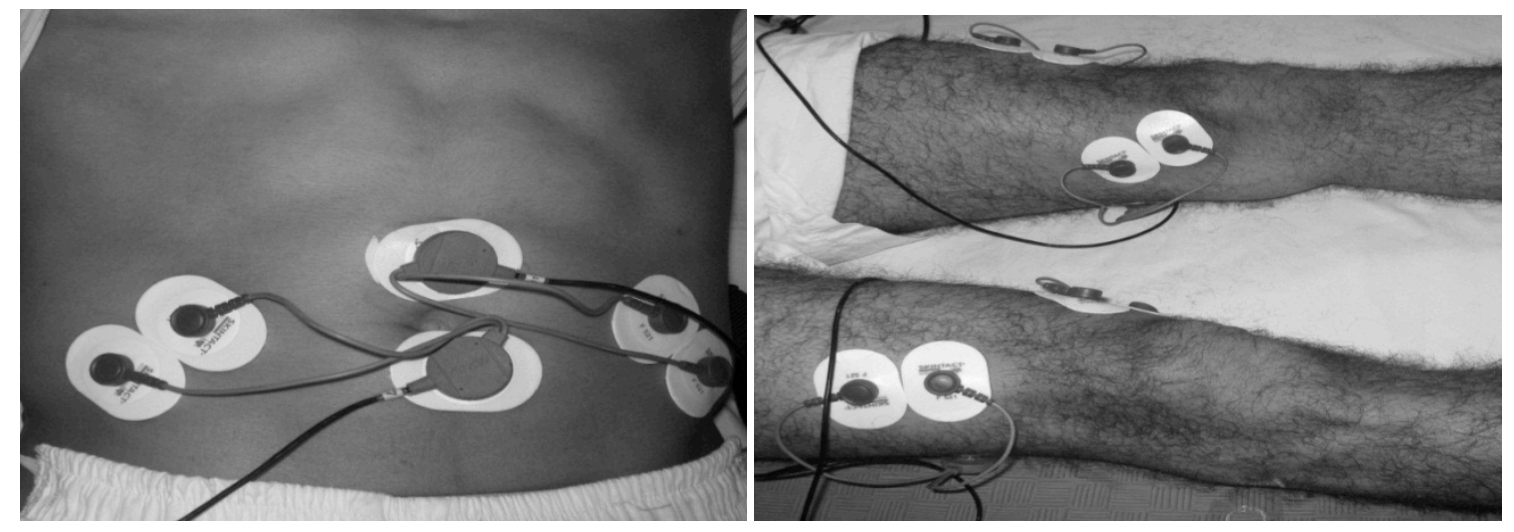

Figure 1. The position of the Chest Leads

The electrogoniameter was placed at the outer part of the knee joint on the external Epicondyle as shown in the figure 2. 


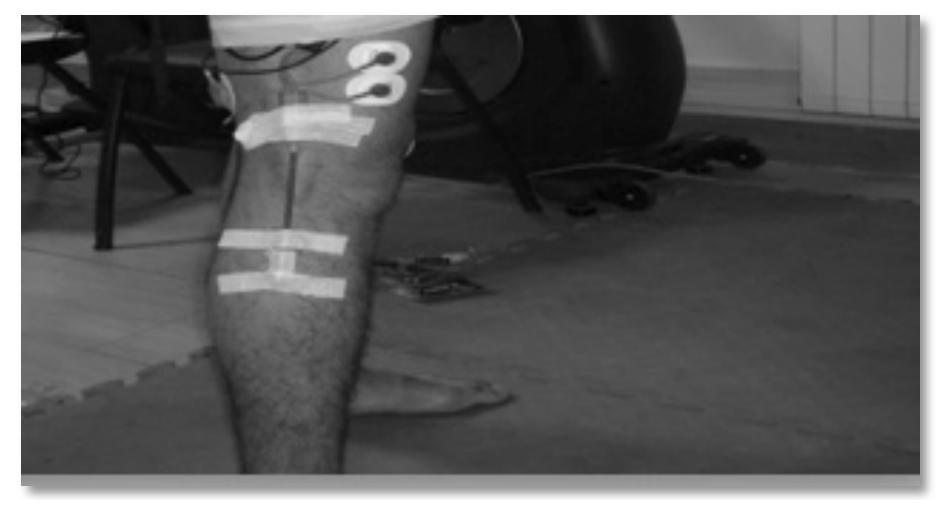

Figure 2. Locating the electrogoniameter

The participants first jogged on the treadmill to warm up for six minutes. Then, they practiced some certain stretching exercises for four minutes which were planned before to prepare the discussed muscles (the type and length of the exercise were specified). After that, the participants with examiner's alert started implementing 3 kicks of Ura Mawashi Geri in a five-second interval with their dominant legs (front leg) on the target kept above the examiners head in an appropriate distance with the participants lower limbs.

It should be noted that the participants had practiced the procedure before the original test for several times in order to get familiar with it. And they performed the technique with the maximum speed at all phases of its implementation.

After processing the data and RMS records, the onset latency of each muscle were measured at any phases by the Mean+4sd approach (Goniometric records). The average of the obtained records was used to determine the onset of each muscle separately at all phases of the implementation of the techniques.

\section{RESULTS}

In this study, the technique, Ura Mawashi Geri was divided into six distinct stages according to the movements of the knee joint (figure 3).

Phase 1: From guarding position of starting the movement to the knee flexion.

Phase 2: From the knee flexion to the knee extension.

Phase 3: From the knee extension to the knee flexion (kicking the target).

Phase 4: From the knee flexion to the knee extension (after the kick).

Phase 5: From the knee extension to the knee flexion.

Phase 6: from the knee flexion to the ultimate knee extension and back to the guarding position. 


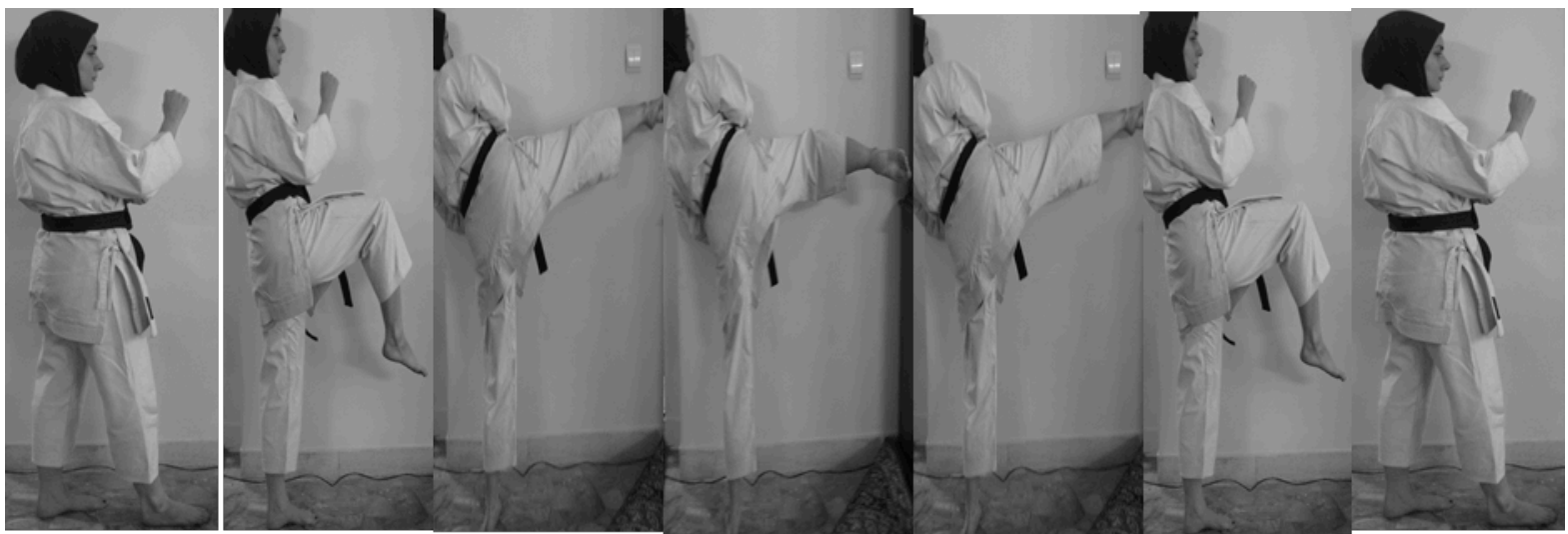

Figure 3. All the phases of implementing the Ura Mawashi Geri technique

The average onset latency of the muscles of VM, VL, GM and EO is presented separately at all phases of the technique. At the first phase the muscles stimulation time pattern is respectively GM, EO, VL and VM, which means that GM as the first muscle involves - 37/39 milliseconds earlier than the VM as the last muscle in this technic.

First Phase

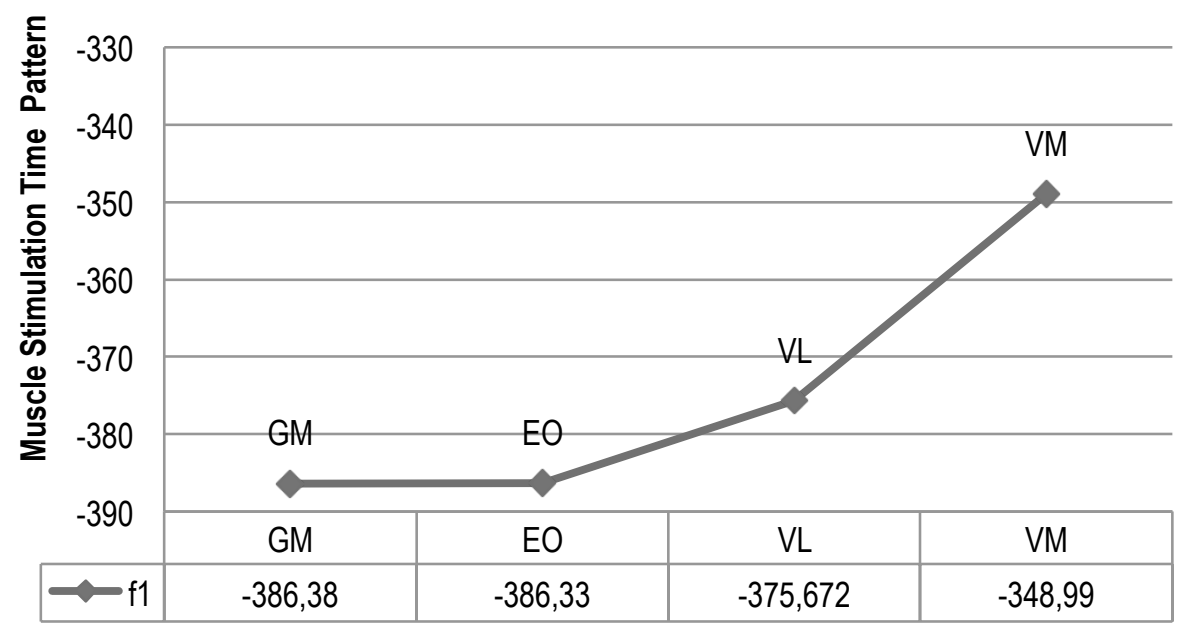

Figure 4. The muscle stimulation time pattern at the first phase of implementing Ura Mawashi Geri technique

Diagaram 2: It illustrates the order of muscles called into action at the second phase of implementing Ura Mawashi Geri which is respectively in the order of VM, GM, EO and VL. 


\section{Second Phase}

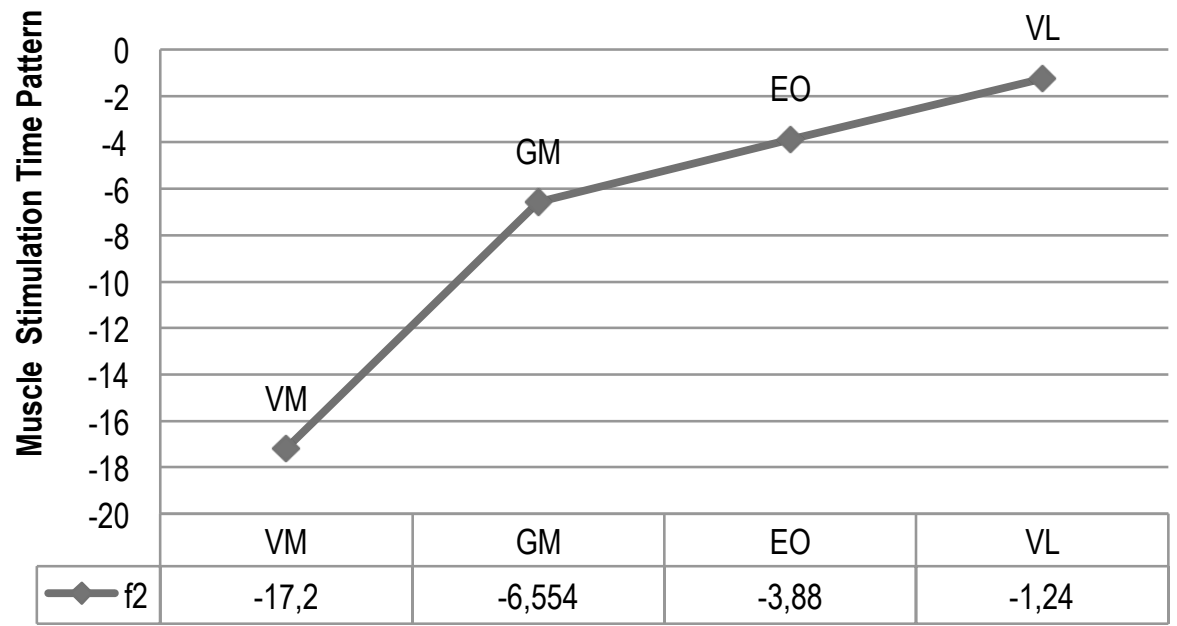

Figure 5. The muscle stimulation time pattern at the second phase of implementing Ura Mawashi Geri technique

In the third diagram, the muscles stimulation time shows a respectively order of EO, VM, GM and VL when implementing the technic it means VL (as the last involving muscle) involves $21 / 3$ milliseconds later than EO (the first involving muscle).

\section{Third Phase}

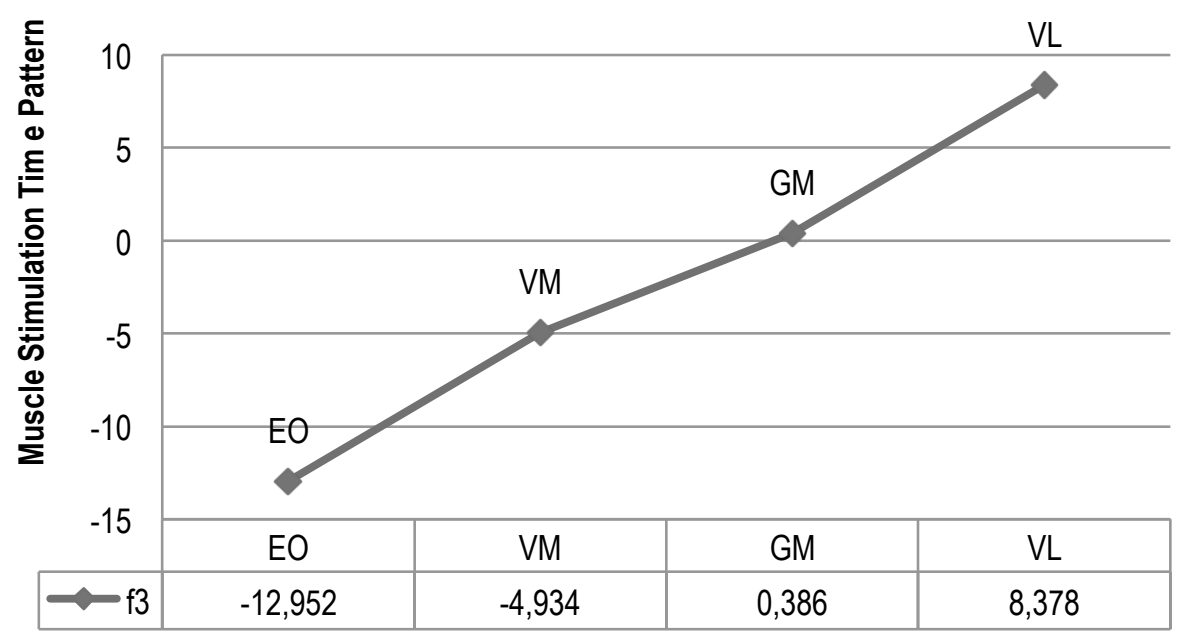

Figure 6. The muscle stimulation time pattern at the third phase of implementing Ura Mawashi Geri technique

As portrayed in diagram 4, the order of the muscle stimulation in the fourth phase of implementing Ura Mawashi Geri was respectively GM, VM, EO and VL. 


\section{Fourth Phase}

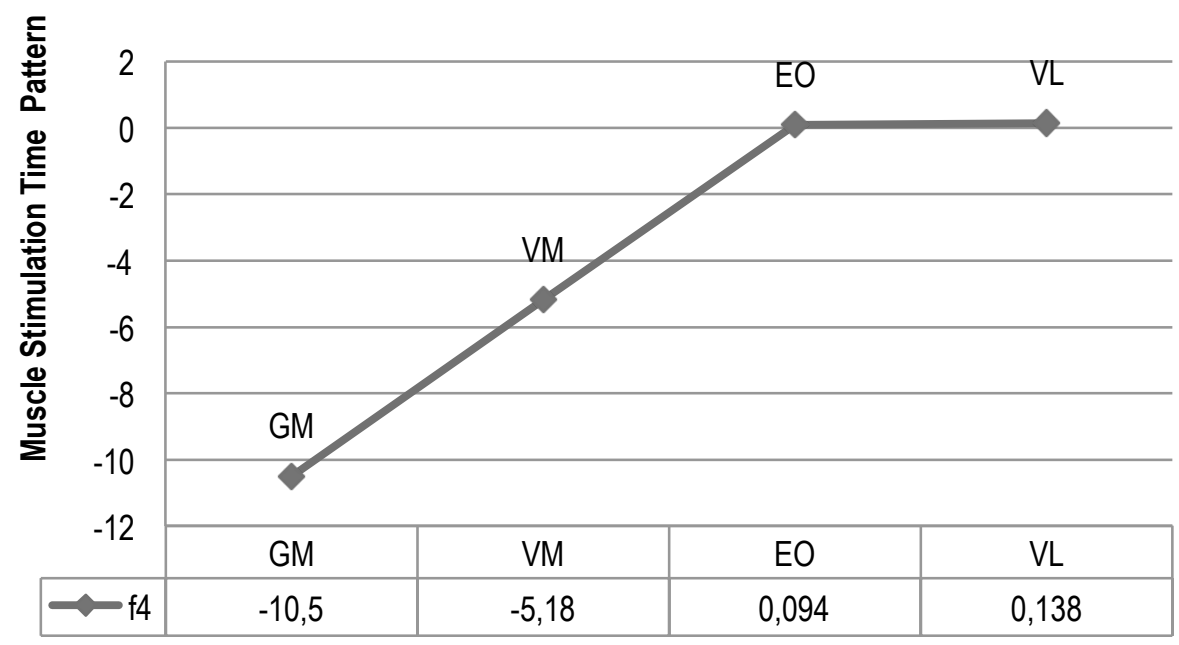

Figure 7. The muscle stimulation time pattern at the fourth phase of implementing Ura Mawashi Geri technique

According to Figure 8 in the fifth phase of implementing Ura Mawashi Geri the muscle involvement order is respectively GM, VM, EO and VL.

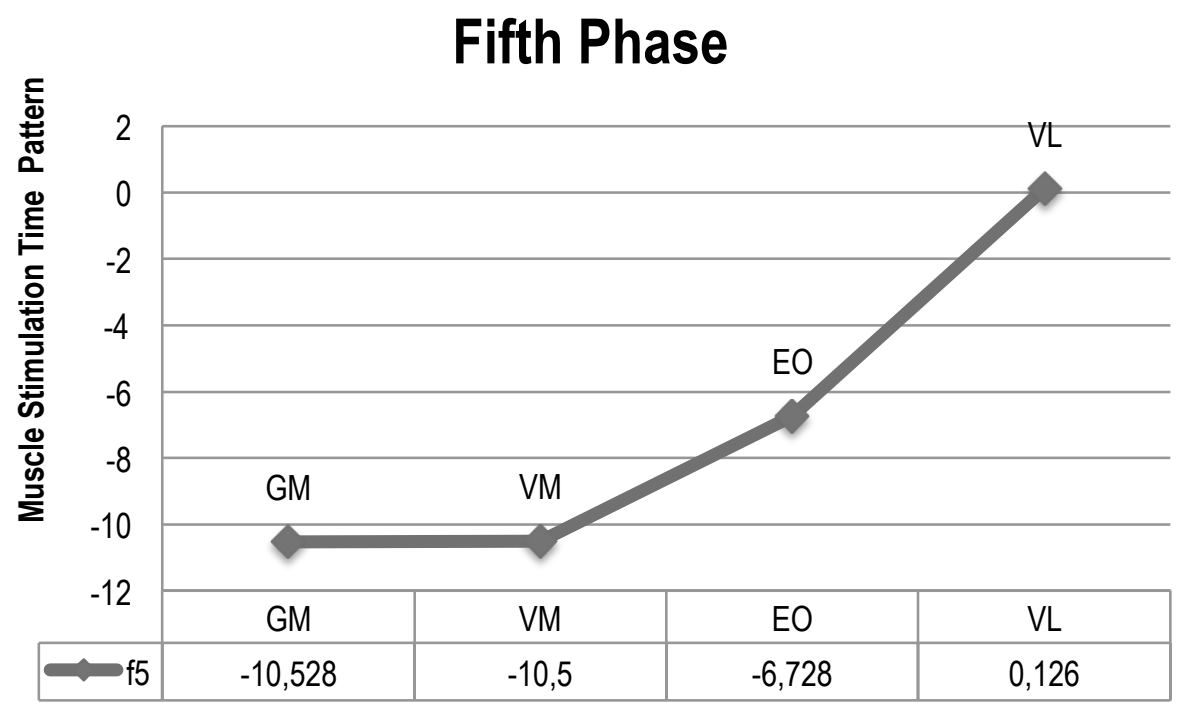

Figure 8. The muscle stimulation time pattern at the fifth phase of implementing Ura Mawashi Geri technique

As the sixth figure shows the sixth phase of the implementation of Ura Mawashi Geri, the muscle stimulation time pattern is respectively VM, VL, GM and EO where VM involves - 5/36 milliseconds earlier than EO. 


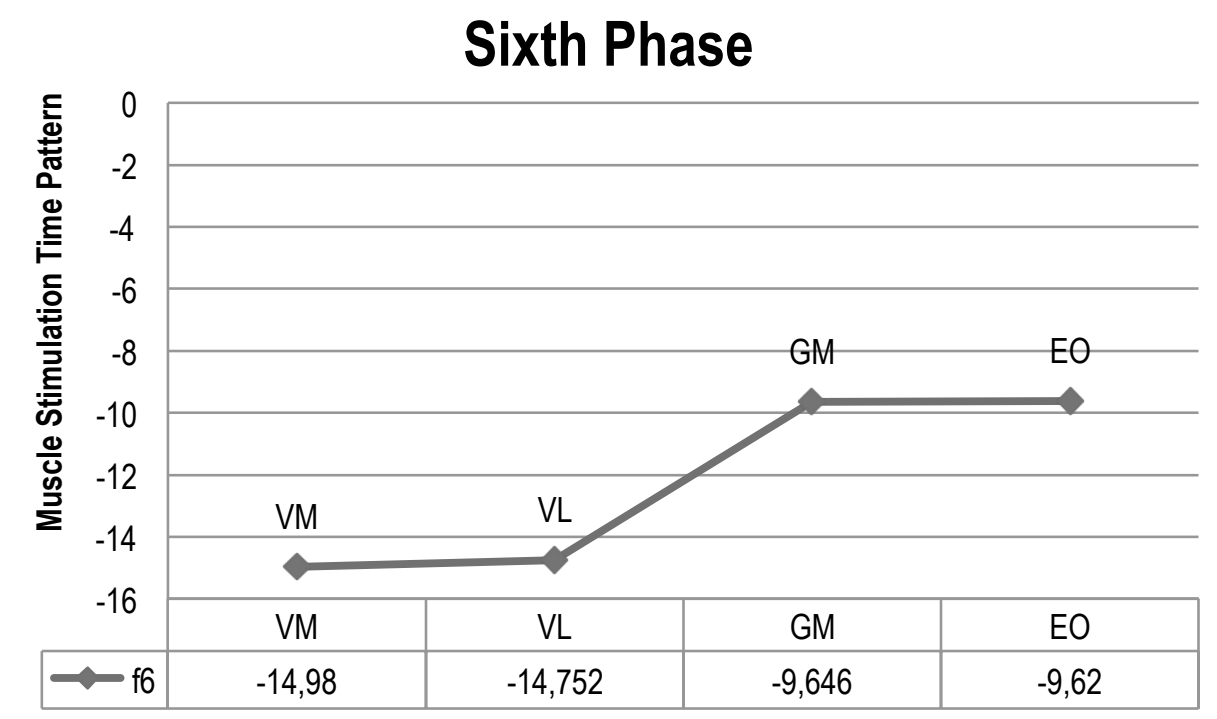

Figure 9. The muscle stimulation time pattern at the sixth phase of implementing Ura Mawashi Geri technique

\section{DISCUSSION AND CONCLUSION}

The purpose of this study is to provide the muscle stimulation time pattern of EO, GM, VL and VM muscles at any of the phases of implementing Ura Mawashi Geri by the dominant leg. Karate is divided into two fields of Kata and Cumite with a wide range of technics using different parts of the body (Critchley \& Meredith, 1999; Emmermacher et al., 2007; Witte et al., 2007; Earl et al., 2001; Mellor, 2005; MacGregor et al., 2005; Stensdotter et al., 2008; Wong, 2006; Mahmoud \& El-Daly, 2010; Antakly, 2010; Leinonen, 2013; Coqueiro et al., 2005; Cavazzuti et al., 2010; Herrington \& Pearson, 2006; Herrington, et al., 2006; Ivkovic et al., 2007; Jafari et al., 2003; Akuthota et al., 2008; Baffal et al., 2012; Sarabon et al., 2010; Wong, 2009; Dixon \& Howe, 2007; Ebrahimi \& Hafezi 2000; Dbbagh et al., 2008). Ura Mawashi Geri is a technic involving different muscles such as VM, VL, EO and GM etc. It is the result of the movements such as flexion, extension, pelvic tilt and the abduction of thigh and knee and the lumbar- pelvic areas. In addition to this, the body weight also transfers in lower limb while performing the technic.

There are lots of reasearches done on the comparison of the onset latency of VM, VL, GM and EO muscles when doing activities such as going up the stairs, explosive actions, Scout and mountain climbing on healthy and injured people which shows these people suffer from Patella Femoral Syndrome in VM and VL. This causes onset latency in LV among people suffering from VM injury (Mellor, 2005; MacGregor et al., 2005; Stensdotter et al., 2008; Wong, 2006; Sarabon et al., 2010; Lederman, 2010; Witte et al., 2008; Camomilla et al., 2009; Ekstrom et al., 2007). There was no research found on the muscle stimulation time pattern of EO, GM, VL and EO when implementing the Ura Mawashi Geri technic at all its phases. At any phases of technic, regarding the different motions of knee, thigh, lumbar- pelvic areas and the trunk, the weight transfer in muscle stimulation time pattern of $\mathrm{EO}, \mathrm{GM}, \mathrm{VL}$ and $\mathrm{VM}$ varies, and according to the order and importance of the movements in each phase, the muscle stimulation timie varies.

The results of this study suggest that in the first, fourth-and fifth phases of implementing Ura Mawashi Geri, the GM muscle involves earlier than the other muscles. At these phases, the side pelvic tilt, knee extension 
and abduction of the GM muscle involve earlier as the stabilizer of the patella joint, lumbar - pelvic areas and also the postural control stabilizer due to weight transfer of body than other muscles (Baffal et al., 2012; Sarabon, et al., 2010; O'Sullivan et al., 2010). At the sixth phase of implementation of Ura Mawashi Geri, it is the VM muscle which involves the earliest due to the knee extension resulting in earlier reaction of this muscle as the stabilizer of Patella joint. At the end of the third phase, when kicking the target, the earlier muscle involving in the action is EO as a result of Anterior Pelvic Tilt (Akuthota et al., 2008).

At the end, it can be concluded that the stability of joints and postural control of body is very important for the precise implementation of Ura Mawashi Geri with the appropriate speed and power. As the results show, the stability muscles in joints involve in the action earlier than the dynamic muscles at their activity phase.

\section{REFERENCES}

1. Critchley, G., \& Meredith, S. (1999). Injury rates in Shotokan karate. Br Journal Sports Medicine, 33(3), pp.177-174.

2. Emmermacher, P., Witte, K., Bystryzycki, S., \& Potenberg, J. (2007). DIFFERENT VARIATIONS OF THE KARATE TECHNIQUE MAWASHI-GERI.

3. Witte, K., Emmermacher, P., Bystryzycki, S., \& Potenberg, J. (2007). MOVEMENT STRUCTURES OF ROUND KICKS IN KARATE.

4. Earl, J.E., Schmitz, R.J., \& Arnold, B.L. (2001). Activation of the VMO and VL during dynamic minisquat exercises with and without isometric hip adduction. Electromyography and Kinesiology, 11(6), pp.381-386.

5. Mellor, H. (2005). Motor unit synchronization between medial and lateral vasti muscles. Clinical Neurophysiology, 116(7), pp.1585-1595.

6. MacGregor, K.S., Gerlach, S., Mellor, R., \& Hodges, P.W. (2005). Cutaneous stimulation from patella tape causes a differential increase in vasti muscle activity in people with patellaofemoral pain. Journal of Orthopaedic Research, 23(2), pp.351-358.

7. Stensdotter, A.K., Grip, H., Hodges, P.W., \& Hager-Ross, C. (2008). Quadriceps activity and movement reactions in response to unpredictable sagittal support-surface translations in women with patellofemoral pain. Journal of Electromyography and Kinesiology, 18(2), pp.298-307.

8. Wong, Y. (2006). Surface electrode placement affects the EMG recordings of the quadriceps muscles. Physical Therapy in Sport, 7(3), pp.122-127.

9. Mahmoud, A., \& El-Daly, S. (2010). Biokinematical Analysis for "Mai - Mawashi - Geri" Based on Two Different Levels of the Competitor's Body in Karate. World Journal of Sport Sciences, 3(3), pp.161-167.

10. Antakly, F. (2010). I.S.K.F. Spotlight, 14; pp.13-15.

11. Leinonen, H. (2013). Are martial arts good for your core strength? IQ Journal, 2(1), pp.35-42.

12. Coqueiro, K., Bérzin, F., Bevilaqua-Grossi, D., Soares, A.B., Candolo, C., \& Monteiro-Pedro, V. (2005). Analysis on the activation of the VMO and VLL muscles during semisquat exercises with and without hip adduction in individuals with patellofemoral pain syndrome. Journal of Electromyography and Kinesiology, 15(6), pp.596-60.

13. Cavazzuti, L., Merlo, A., Orlandi, F., \& Campanini, I. (2010). Delayed onset of electromyographic activity of vastus medialis obliquus relative to vastus lateralis in subjects with patellofemoral pain syndrome. Gait \& Posture, 82(2), pp.290-295. 
14. Herrington, L., \& Pearson, S. (2006). Does level of load affect relative activation levels of vastus medialis oblique and vastus laterialis? Journal of Electromyography and Kinesiology, 16(4), pp.379-383.

15. Herrington, L., Blacker, M., Enjuanes, N., Smith, P., \& Worthington, D. (2006). The effect of limb position, exercise mode and contraction type on overall activity of VMO and VL. Physical Therapy in Sport, 7(2), pp.87-92.

16. Ivkovic, A., Franić, M., Bojanić, I., \& Pećina, M. (2007). Overuse Injuries in Female Athletes. Medicine Journal, 48(6), pp.767-772.

17. Jafari, H., Shah Hosseini, G.R., Ebrahimi, E., \& Shaterzadeh. M.J. (2003). The Analysis of timing and level of the electrical activity of the muscles around the knee joint in reactionary active patterns of movements. Iran University of Medical Sciences, 10(35), pp.361-372

18. Akuthota, A., Ferreiro, F., Moore, T., \& Fredericson, M. (2008). Core Stability Exercise Principles. The American College of Sports Medicine, 7(1) pp.39-44.

19. Baffal, A., Felicio, L., Saad, M.C., Nogueira-Barbosa, M.H., Santos, A.C., \& Bevilaqua-Grossi, D. (2012). Quantitative MRI of Vastus Medialis, Vastus Lateralis and Gluteus Medius Muscle Workload after Squat Exercise: Comparison Between Squatting with Hip Adduction and Hip Abduction. Journal of Human Kinetics volume, 33, pp.5-14.

20. Sarabon, N., Polona, P., \& Burggraf, S. (2010). Proposal for progressive loading of the hip abductors under mechanically unstable conditions: An electromyography study. European Journal Translational Myology - Basic Applied Myology, 20, pp.187-192.

21. Wong, Y. (2009). Recording the vastii muscle onset timing as a diagnostic parameter for patellofemoral pain syndrome: fact or fad? Physical Therapy in Sport, 10(2), pp.71-74.

22. Lederman, E. (2010). The myth of core stability. Journal of Bodywork \& Movement Therapies, 14(1), pp.84-98.

23. Dixon, J., \& Howe, T. (2007). Activation of vastus medialis oblique is not delayed in patients with osteoarthritis of the knee compared to asymptomatic participants during open kinetic chain activities. Manual Therapy, 12(3), pp.219-225.

24. Ebrahimi Takamjani, E., \& Hafezi, R. (2000). The effect of the different knee angles in two open and closed kinematic chains on the ratio of the electrical activity of the muscles of Vastus Medialis Oblique (vmo) to Vastus Lateralis (vl) during the isometric contraction. Journal of Iran University of Medical Sciences, pp.73-80.

25. Dbbagh Nikoo Kheslat, S., Shahbazi Moghadam, M., \& Sheykh, M. (2008). Investigation of the kinetics chain punching in karate. Journal of Harakat ( movement), pp.97-107

26. Witte, K., Emmermacher, P., \& Lessau, Marion. (2008). Biomechanical Measuring Stations To Solve Problems in karate sport. Coaching and Sports Performance, 1(1), pp.565-568.

27. Camomilla, V., Sbriccoli, P., Quinzi, F., Bergamini, E., Mario, A., \& Felici, F. (2009). RoundHouse Kick With And Without Impact In Karateka Of Different Technical Level. 27 International Conference on Biomechanics in Sports.

28. Ekstrom, R,. Donatelli, \& Carp, K.C. (2007). Electromyographic Analysis of Core Trunk, Hip, and Thigh Muscles During 9 Rehabilitation Exercises. Journal of Orthopaedic \& Sports Physical Therapy, 37(12), pp.754-762.

29. O'Sullivan, K., Smith, S.M., \& Sainsbury, D. (2010). Electormyographic analysis of the three subdivisions of gluteus medius during weight-bearing exercises. Journal o Sports Medicine a Rehabilitation Teerapy Technology, 12(2), pp.2-17. 